\title{
POEMS SYNDROME ALTHOUGH RARE PRESENTS A CONSTELLATION OF CLINICAL FINDINGS THAT ALLOWS ITS EARLY DIAGNOSIS
}

Gabriela da Silva Cremonese ${ }^{1, \star}$, Ivânio Alves Pereira ${ }^{1}$

1.Universidade do Sul de Santa Catarina, Palhoça (SC), Brazil.

*Corresponding author: gabrielacremonese@gmail.com

\section{BACKGROUND}

Polyneuropathy, organomegaly, endocrinopathy, monoclonal protein, skin changes (POEMS) syndrome is defined by the acronym composed by the set of clinical findings: polyneuropathy, organomegaly, endocrinopathy, monoclonal protein and skin changes. This is a paraneoplastic syndrome secondary to dysplasia of plasma cells which quality and rarity and chronic evolution become its diagnosis and challenge, especially in recent cases of the disease. The authors describe a case of POEMS in a 55-yearold patient with clinical symptoms for more than three years and discuss a constellation of classic clinical findings that could have allowed or recognized in the most initial phase.

\section{CASE REPORT}

Tree years ago, a 55-year-old male patient presented acrocyanosis associated with a severe pain in the hands and glove and stocking paraesthesia, besides walking difficulties. During this period, he had a weight loss of $30 \mathrm{~kg}$ and asthenia. In the clinical examination, in addition to acrocyanosis, recent telangiectasias begun on the trunk and face, bilateral gynecomastia and palpable hepatomegaly were found $4 \mathrm{~cm}$ below the right costal margin. The neurological examination detected global hyporeflexia, global distal motor strength grade $4 / 5$ on the extremities, reduced painful sensitivity in lower limbs and loss of vibration sense in upper limbs and lower limbs. He had polycythemia and a monoclonal peak between beta 2 and gamma globulin fractions. Electromyography showed polyneuropathy with a chronic inflammatory demyelinating pattern. Moreover, osteosclerotic and lytic areas permeating the body of the sternum and skull osteosclerotic lesions were revealed by computed tomography scans. Thus, a bone marrow aspirate revealed $9 \%$ of plasma cells and sternal biopsy, besides a bone marrow hypocellularity with mild polyclonal reactive plasmacytosis. The set of such findings allowed the final diagnosis of POEMS Syndrome, an osteosclerotic multiple myeloma. The treatment was based on cycles of dexamethasone, disodium pamidronate, amitriptyline and gabapentin for the management of neuropathic pain, followed by a VTD protocol (bortezomib + doxorubicin + thalidomide).

\section{CONCLUSION}

Even though it is a rare disease, rheumatologists and general practitioners must include the POEMS Syndrome on differential diagnosis of diseases, such as polyneuropathies, osteosclerotic lesions, plasma cell dyscrasias, neoplasms, infections and vasculitis. In the face of a patient with this hypothesis, making the detection can be a challenge, but a good history and physical examination (H\&P) in addition to appropriate exams can distinguish this syndrome from its differential diagnoses. 